\title{
MWC 314 - A NEW GALACTIC B[e] SUPERGIANT
}

\author{
ANATOLY S. MIROSHNICHENKO \\ Central Astronomical Observatory of the Russian Academy of Sciences at Pulkovo, \\ 196140 Saint-Petersburg, Russia, e-mail:anat@gaoran.spb.su
}

We present a new study of MWC $314=\mathrm{BD}+14^{\circ} 3887$ - a poorly investigated object with strong emission lines and IR excesses. Merrill (1927) payed attention to it because of the presence of hydrogen and Fe II emissions in its spectrum. Swensson (1942) also detected interstellar lines $\mathrm{H}$ and $\mathrm{K} \mathrm{Ca}$ II and $4430 \AA$ band, Balmer emissions from $H_{\alpha}$ to $H_{8}, \mathrm{NaI} 5890$ and $5896 \AA$ emissions and estimated its spectral type as gG2-3 or dG4-5 from the SED in continuum, and B2 from the excitation degree. Photospheric lines and spectral features of late-type stars were not observed. Allen (1973) noted that the object's SED corresponds to that of a late-type star but it might be a symbiotic system or a reddened normal star. The IRAS fluxes were obtained only at 12 and $25 \mu \mathrm{m}$. The object is unknown as a radio source. From this we can conclude that this system consists of, at least, a hot star surrounded by a gaseous envelope.

We obtained 7 UBVRIJHK and 25 UBVRI observations at the 1-m telescope of the Astrophysical Institute of Kazakhstan Academy of Sciences with the two-channel photometer - polarimeter (Bergner et al., 1988) in 1989-1993 (diaphragm $26^{\prime \prime}$ ). We have detected $\sim 0 \stackrel{\mathrm{m}}{3}$ variability in all photometric bands. The changes in the optical are synchronous in general. A Fourier analysis of the optical light curves, using Deeming's (1975) technique, show that we can discriminate a regular variability with a period of 4.16 . Phase diagrams are most clear in the B and $\mathrm{V}$ bands (the amplitudes are 0.25 and 0.15 respectively). The spectra were obtained in July 1991 at the 6-m telescope of the Special Astrophysical Observatory at the Northern Caucasus with a photoelectric scanner in the range of $4000-7400 \AA$ and a dispersion of $50 \AA / \mathrm{mm}$.

The profiles of hydrogen emissions are symmetric without $\mathrm{P}$ Cyg-type absorption. The Balmer decrement is $I_{\mathrm{H}_{\alpha}}: I_{\mathrm{H}_{\beta}}: I_{\mathrm{H}_{\gamma}}=1: 0.060: 0.0011$. We have also detected some absorptions which we suppose to be photospheric. These lines together with the weak He I emissions imply that the underlying star is not later than B2. There is no evidence for circumstellar dust radiation between 1 and $25 \mu \mathrm{m}$. We have used Lamers and Waters'(1984) method to fit the SED and have found the best fit with the following parameter values: $A_{\mathrm{V}}=5{ }^{\mathrm{m}} 5$, spectral type - $\mathrm{B} 1\left(T_{*}=23500 \mathrm{~K}\right)$, wind temperature $T=T_{*}$. A comparison of the dereddened Balmer decrement with numerical results for spherical decelerating stellar winds obtained by Pogodin's(1986) method have given the following values of electron density number near the 
photosphere and volume emission measure: $\log N_{\mathrm{eo}}=11.5$ and $\log \varepsilon \sim 60.5$. It is more difficult to determine the star's mass or radius because we do not know its distance (D). An upper limit of the star's radius can be estimated from the suggestion that the regular variability is connected with the region of the envelope very close to the photosphere, because more clear phase pictures are observed in the bands where the envelope's influence is smallest. Hence we can use the Kepler's third law. Together with the expression for bolometric luminosity it gives the relation between $\mathrm{L}$ and $M_{*}$. Comparing it with the evolutionary tracks (Maeder and Meynet, 1987) we obtaine the best agreement between them for $\log \left(L / L_{\odot}\right)=5.5, M \sim 30 M_{\odot}$, and an upper limit of $R_{*}=35 R_{\odot}$. The characteristics of $H_{\alpha}, \varepsilon$, and the parameters of the SED fitting give values of the star's radius near $30 R_{\odot}$ and a mass-loss rate of about $10^{-6} M_{\odot} / \mathrm{yr}$. Thus we can conclude that MWC 314 is a supergiant. Supposing $R_{*}=30 R_{\odot}$, the distance towards the object is about $1.7 \mathrm{kpc}$, and the radio flux at $5 \mathrm{GHz} \sim 6 \mathrm{mJy}$.

It is difficult to determine the age of MWC 314 because the tracks for stars in this part of HR diagram are almost parallel. Its characteristics are close to that of LBVs and $\mathrm{B}[\mathrm{e}]$ supergiants, but the photometric behaviour has not been studied long enough to make a comparison. We know that most of $B[e]$ supergiants have circumstellar dust, and it is suggested that they are transition objects between Of and WR stars (Zickgraf et al., 1986). It has been unclear in which evolutionary stage massive stars can have dust shells. So, MWC 314 may be an object in a stage before or after the dust formation process.

\section{References}

Allen D.A., MNRAS, 1973, 161, 145

Bergner Yu.K., Bondarenko S.L., Miroshnichenko A.S. et al., Izvestia Glavn. Astron. Obs. v Pulkove, 1988, 205, 142, (in Russian)

Deeming T.J., Ap\&SS., 1975, 36, 137

Lamers H.J.G.L.M. and Waters L.B.F.M., A\&A, 1984, 136, 37

Maeder A. and Meynet G., A\&A, 1987, 182, 243

Merrill P.W., ApJ, 1927, 65, 286

Pogodin M.A., Astrofizika, 1986, 24, 491 (in Russian)

Swennson J.W., ApJ, 1942, 97, 226

Zickgraf F.-J., Wolf B., Stahl O., Leitherer C., Appenzeller I., 1986, A\&A, 163, 119 